\title{
Erratum to: TREK: an integrated system architecture for intraoperative cone-beam CT-guided surgery
}

\author{
A. Uneri - S. Schafer - D. J. Mirota - S. Nithiananthan - Y. Otake • \\ R. H. Taylor - G. L. Gallia - A. J. Khanna - S. Lee - D. D. Reh • \\ J. H. Siewerdsen
}

Published online: 7 August 2011

(C) CARS 2011

\section{Erratum to: Int J CARS \\ DOI 10.1007/s11548-011-0636-7}

Unfortunately, several authors were omitted in the original version of the article. The missing authors and their affiliations are given below:

The online version of the original article can be found under doi:10.1007/s11548-011-0636-7.

\footnotetext{
A. Uneri

Department of Computer Science, Johns Hopkins University,

Traylor Building, Room \#726, 720 Rutland Avenue,

Baltimore, MD 21205-2109, USA

e-mail: ali.uneri@jhu.edu

S. Schafer $\cdot$ S. Nithiananthan

Department of Biomedical Engineering, Johns Hopkins University,

Traylor Building, Room \#726, 720 Rutland Avenue,

Baltimore, MD 21205-2109, USA

e-mail: sebastian.schafer@jhu.edu

S. Nithiananthan

e-mail: sajendra@jhu.edu

D. J. Mirota

Department of Computer Science,

Johns Hopkins University, 224 NEB,

3400 N. Charles Street,

NE Baltimore, MD 21218, USA

e-mail: dan@cs.jhu.edu

\section{Y. Otake}

Department of Computer Science, Johns Hopkins University, 128 Hackerman Hall, 3400 N. Charles Street, NE Baltimore, MD 21218, USA

e-mail: otake@jhu.edu

R. H. Taylor

Department of Computer Science, Johns Hopkins University, 127 Hackerman Hall, 3400 N. Charles Street, NE Baltimore, MD 21218, USA

e-mail: rht@jhu.edu
}

The correct order of authors is:

A. Uneri · S. Schafer · D. J. Mirota $\cdot$ S. Nithiananthan . Y. Otake · R. H. Taylor · G. L. Gallia · A. J. Khanna . S. Lee · D. D. Reh · J. H. Siewerdsen

G. L. Gallia

Department of Neurosurgery, The Johns Hopkins Hospital,

Phipps Building, Room 118, 600 N. Wolfe Street,

Baltimore, MD 21287, USA

e-mail: ggallia1@jhmi.edu

\section{A. J. Khanna}

Department of Orthopaedic Surgery, Johns Hopkins University, Good Samaritan Hospital, 5601 Loch Raven Blvd., Room G-1, Baltimore, MD 21239, USA

e-mail: akhanna1@jhmi.edu

S. Lee · D. D. Reh

Department of Otolaryngology, Head and Neck Surgery,

Division of Rhinology and Sinus Surgery,

Johns Hopkins University, Johns Hopkins University JHOC,

6th Floor, 601 N. Caroline Street, Baltimore,

MD 21287-0910, USA

e-mail: slee39@jhmi.edu

D. D. Reh

e-mail: dreh1@jhmi.edu

J. H. Siewerdsen

Department of Computer Science, Johns Hopkins University, Baltimore, MD, USA

\section{J. H. Siewerdsen $(\varangle)$}

Department of Biomedical Engineering, Johns Hopkins University, Traylor Building, Room \#718, 720 Rutland Avenue, Baltimore,

MD 21205-2109, USA

e-mail: jeff.siewerdsen@jhu.edu 